\title{
Effect of Overnight Staining on the Quality of Flow Cytometric Sorted Stallion Sperm: Comparison with Tradtitional Protocols
}

\author{
C. M. Balao da Silva, C. Ortega Ferrusola, J. M. Gallardo Bolanos, M. Plaza Davila, P. \\ Martin-Munoz, J. M. Morrell, Heriberto Rodriguez-Martinez and F. J. Pena
}

\section{Linköping University Post Print}

\section{Tweet}

N.B.: When citing this work, cite the original article.

Original Publication:

C. M. Balao da Silva, C. Ortega Ferrusola, J. M. Gallardo Bolanos, M. Plaza Davila, P. MartinMunoz, J. M. Morrell, Heriberto Rodriguez-Martinez and F. J. Pena, Effect of Overnight Staining on the Quality of Flow Cytometric Sorted Stallion Sperm: Comparison with Tradtitional Protocols, 2014, Reproduction in domestic animals (1990), (49), 6, 1021-1027. http://dx.doi.org/10.1111/rda.12431

Copyright: Wiley: 12 months http://eu.wiley.com/WileyCDA/

Postprint available at: Linköping University Electronic Press http://urn.kb.se/resolve?urn=urn:nbn:se:liu:diva-113053 


\title{
Effect of overnight staining on the quality of sex sorted stallion sperm: comparison with the traditional protocol
}

Balao da Silva $\mathrm{CM}^{l}$, Ortega Ferrusola $\mathrm{C}^{l}$, Gallardo Bolaños, $\mathrm{JM}^{l}$, Plaza Dávila $\mathrm{M}^{l}$, MartínMuñoz P, Morrell $\mathrm{JM}^{3}$, Rodriguez Martínez $\mathrm{H}^{4}$, Peña $\mathrm{FJ}^{l^{*}}$.

${ }^{1}$ Laboratory of Equine Reproduction and Equine Spermatology. Veterinary Teaching Hospital, ${ }^{3}$ Division of Reproduction, Faculty of Veterinary Medicine and Animal Sciences, Swedish University of Agricultural Sciences, Uppsala, Sweden ${ }^{4}$ Department of Clinical and Experimental Medicine, Faculty of Health Sciences, Linköping University, Sweden

\section{Acknowledgements}

The authors received financial support for this study from Ministerio de Economía y Competitividad-FEDER in Madrid, Spain, grants AGL 2010-20758 (GAN), AGL2013-43211$\mathrm{R}$ and Gobierno de Extremadura-FEDER (GR 10010 and PCE1002).

\begin{abstract}
Separation of $\mathrm{X}$ and $\mathrm{Y}$-chromosome bearing spermatozoa on the equine is accomplished by flow cytometry. The MoFlo SX DP sorter is highly efficient, allowing the production of foals of the desired sex. However to achieve acceptable pregnancy rates the currently used protocol requires working with fresh semen obtained close or at the sorting facility. An alternative protocol was tested during two consecutive breeding seasons. Fresh stallion semen was cooled for 20h, during which staining with Hoechst 33342 took place. On the following day this sample was flow-sorted and compared with spermatozoa from the same ejaculate that had been sexed on the previous day. All the spermatic parameters evaluated remained unchanged when comparing fresh sorted and refrigerated sorted semen. Pre-sorting storage at $4^{\circ} \mathrm{C}$ does not alter sperm velocities and kinetics, viability and membrane permeability, production of reactive oxygen species, mitochondrial membrane potential and DNA fragmentation index of the sorted sample. This opens the possibility of stallions to be housed far from the sorting facilities. Processed and stained sperm could be shipped refrigerated on the previous day, sorted and inseminated on the next.
\end{abstract}

Keywords: spermatozoa; equine; refrigeration; Hoechst 33342; sex sorting,

\section{Introduction}

Over the last years, sex sorting of stallion spermatozoa has been the subject of growing interest. Owners are relying on ART techniques to improve the benefits while lowering the costs of the production of the best foals, and sorting has become a valuable method (Maxwell, Evans et al. 2004). However, since freezing of sex-sorted stallion spermatozoa withholds low fertility results (Clulow, Buss et al. 2008), and equine semen presents an enormous individual variability in their response to cryopreservation (Vidament 2005), creating an alternative protocol appear appropriate. Post-sorting storage has already been tested on boar spermatozoa. Apparently, $5 \mathrm{~h}$ at $20^{\circ} \mathrm{C}$ is the maximum time during which sorted semen could be stored, in order to maintain the fertilizing ability (Parrilla, Vazquez et al. 2005). Indeed, similar data was verified in Italy, where liquid storage of sex-sorted sperm for 24 to $26 \mathrm{~h}$ at $15^{\circ} \mathrm{C}$ significantly decreased the fertility results (Spinaci, Vallorani et al. 2010). Given the high susceptibility that stallion spermatozoa have to the sorting procedure (Gibb, Butler et al. 2013), we considered testing a pre-sorting storage. The sorting procedure includes chemical and physical damages to spermatozoa, among them staining and incubation with Hoechst 33342, high pressures, U.V light exposure, electrical charge, mechanical forces, dilution and re-concentration post sorting (Maxwell and Johnson 1999; Suh, Schenk et al. 2005). These damages are referred to reduce 
lifespan, leading to a decrease on the fertilizing ability of sorted spermatozoa (Maxwell, Evans et al. 2004). As stated by others, mechanical damage is apparently one of the most important damages induced by the technique (Garner 2001). Therefore, it is possible that the damage imposed by the overnight incubation in Hoechst 33342 does not suppose significant differences on the spermatic parameters.

The consequences of the damages caused by this technique are currently being studied. Previous findings include destabilization of the plasmalemma after the sorting procedure (Clulow, Evans et al. 2010; Balao da Silva, Spinaci et al. 2013), which probably permeabilizes the plasmatic membrane, allowing passage and or release of molecules. The relation between membrane permeabilization, induction of apoptosis and oxidative stress has also been established (Balao da Silva, Ortega Ferrusola et al. 2013). On the other hand, the main effects of cooling stallion sperm for 2-4 days are on motility, membrane permeability and apoptotic-like phenomena, (Gallardo Bolanos, Miro Moran et al. 2012). Although both techniques (flow-cytometric sorting and cooling) are probably altering the plasmalemma and the intracellular homeostasis of spermatozoa, we placed the hypothesis that a reduced refrigeration period that includes staining of DNA, prior to sorting, could be feasible. On a practical application of this hypothesis, the success of this new protocol would allow a physical separation of the stallion and the sorting facility. It would open the possibility of sending refrigerated stallion semen, previously stained, which would save time at the sorting facility and allow mares to be inseminated close to ovulation (Panarace et al, 2013).

Based on this knowledge, we decided to compare fresh stallion semen sorted after incubation with Hoechst 33342, and sort a sample of the same ejaculate, stained and refrigerated at $5^{\circ} \mathrm{C}$ for $20 \mathrm{~h}$. The quality of the samples was evaluated with a range of different tests, analyzing the oxidative status of the cells, as well as the presence of genomic damage.

\section{Materials and methods}

\section{Experimental design}

The parameters evaluated were: motility and velocity, viability and integrity of the plasmatic membrane, presence of reactive oxygen species (ROS), mitochondrial membrane potential and DNA fragmentation index by SCSA. All the parameters were determined before and after sexsorting on the same day (CONTROL-1 and SEX-1) and on the following day (CONTROL-2 and SEX-2). Experiments were reviewed and approved by the Ethical committee of the University of Extremadura, Spain.

\section{Semen collection and processing}

Semen was obtained from one quarter horse, four Pure Spanish (PRE) horses, one AngloArabian horse, one Lusitanian horse, each of them individually housed at the Veterinary Teaching Hospital of the University of Extremadura, Cáceres, Spain. All individuals were of proved fertility, and maintained according to institutional and European regulations. Semen collection was performed regularly, with a media of two to three collections per week, during the 2012 and the 2013 breeding seasons.

Stallion penis was washed with warm water to reduce contamination, and thoroughly dried. All the ejaculates were collected with a Missouri model artificial vagina, filled with nonspermicidal lubricant and warmed to $45-50^{\circ} \mathrm{C}$, to which an inline nylon micromesh filter was added in order to separate both debris and the gel fraction. Semen was immediately transported to the laboratory for evaluation and processing. Each filtered ejaculate was then extended $1+0.5$ (v/v) in INRA96 (IMV, L'Aigle, France)-Tyrodes (Balao da Silva, Macias-Garcia et al. 2012). All products were bought from Sigma-Aldrich Corporation, St. Louis, MO, USA or Panreac, Barcelona, Spain, and $\mathrm{pH}$ adjusted to 7.2. Four tubes were prepared, with $10 \mathrm{~mL}$ of EquiPure bottom layer (Nidacon Molndal, Sweden) and $10 \mathrm{~mL}$ of EquiPure top layer. To each, $10 \mathrm{~mL}$ of diluted semen were added, regardless of its concentration. Centrifugation was performed, at room temperature during 25 minutes, at 300xg. The resulting sperm pellet was re-extended in 
INRA-T to a final concentration of $100 \times 10^{6} \mathrm{spz} / \mathrm{mL}$. The sperm obtained after colloidal centrifugation was split in four aliquots of 4mL (CONTROL-1, CONTROL-2, SEX-1 and SEX2). To each aliquot, $6 \mu \mathrm{L}$ of Hoechst $33342(16,2 \mathrm{mM})$ was added. The sample CONTROL-1 was left at room temperature throughout the sorting process, filtered with a mesh of $30 \mu \mathrm{m}$, and analyzed. To the sample SEX-1, $4 \mu \mathrm{L}$ of food dye $(0.002 \% \mathrm{wt} / \mathrm{vol}$; FD\&C no. 40 ; Warner Jenkinson Company Inc., St. Louis, MO, USA) was added. This sample was incubated during 90 minutes at $34^{\circ} \mathrm{C}$, sorted and analyzed. The remaining two samples were cooled gradually to $4^{\circ} \mathrm{C}$, by placing the tubes in the freezer in a container filled with water at room temperature. On the following day (after 20 hours of refrigeration) samples were then removed from the freezer and let gradually warm at room temperature. The sample SEX-2 was mixed with $4 \mu \mathrm{L}$ of food dye, let to stabilize during 30 minutes, sorted and analyzed at the same time as a control aliquot (CONTROL-2). All samples were sorted using a MoFlo SX DP (Beckman Coulter Inc., Miami, FL, USA), operating at $40 \mathrm{psi}$ and equipped with an UV argon laser (355nm at $350 \mathrm{~mW}$ ). Sperm were selected using "bulk" sorting, that is, electing both subpopulations of $\mathrm{X}$ and $\mathrm{Y}$ chromosome bearing spermatozoa. Samples were sorted using PBS Puraflo 8x sheath fluid (Beckman Coulter Inc., Miami, Fl, USA). Catch fluid was composed of $2 \mathrm{~mL}$ of INRA-T with $4 \%$ of centrifuged egg yolk (1000 x g for 10 minutes) (Balao da Silva, Macias-Garcia et al. 2012). Obtained sorted sperm suspensions were then centrifuged at 800xg during 20 minutes in order to re-concentrate and analyze the sample.

\section{Sperm motility analysis}

Motility was measured using a computer-assisted sperm analysis system (CASA System, ISAS ${ }^{\circledR}$ Proiser, Valencia, Spain), based on the examination of 25 consecutive, digitalized images obtained from a single field using a 10x negative phase contrast objective in a light microscope (Olympus CX41, Tokyo, Japan). Images were taken with a time lapse of 1s, and the image capture speed was of one every $40 \mathrm{~ms}$. The number of objects incorrectly identified as spermatozoa was minimized on the monitor by using the playback function. Regarding the setting parameters of the program, a spermatozoa was considered immotile when presenting a $\mathrm{VAP}<10 \mu \mathrm{m} / \mathrm{s}$, and motile if it was $>15 \mu \mathrm{m} / \mathrm{s}$. Cells which deviated $<45 \%$ from a straight line were designed as linearly motile.

\section{Flow cytometry}

The cytometric analysis of the spermatic plasmalemma and production of ROS was performed with a MACSQuant Analyzer 10 (Miltenyi Biotec, Bergisch Gladbach, Germany) with three lasers: 405, 488 and $635 \mathrm{~nm}$. Corresponding photomultiplier tubes (PMT) are V1 (Ex $405 \mathrm{Em}$ 450/50), V2 (Ex 405 Em 525/50), B1 (Ex 488 Em 525/50), B2 (Ex 488 Em 585/40), B3 (Ex 488 Em 655-730 655LP + split 730), B4 (Ex 488 Em 750 LP), R1 (Ex 635 Em 655-730 655LP + split 730), R2 (Ex 635 Em 750 LP). Hoechst 33342 was detected by V1, YO-PRO-1 by B1, Ethidium Homodimer-1 by B3 and CellROX by R1. A total of 10000 events per sample were recorded, regarding both forward and sidescatter, and placing a gate of the sperm population with Hoechst 33342 staining, which eliminates non-sperm events. Flow rate was maintained at 200-300 cells/sec. The system is equipped with the MACSQuantify software, creating quadrants that allow the quantification of each subpopulation's frequency. The equipment was calibrated daily with calibration beads provided by the manufacturer and compensation overlap performed before each particular experiment.

Flow cytometric analysis of both mitochondrial membrane potential and SCSA were performed with a Coulter EPICS XL (Coulter Corporation Inc., Miami, FL, USA) at $15 \mathrm{~mW}$, at $488 \mathrm{~nm}$, analyzed by the EXPO 2000 software. For the mitochondrial membrane potential evaluation, subpopulations were divided by quadrants, gated by the analysis of an unstained sample, permitting the frequency quantification of each of the four subpopulations. Non-sperm events were gated out based on the forward scatter and side scatter dot plot by drawing a region enclosing the cell population of interest. Forward and sideways light scatter were recorded for a 
total of 10000 events per sample, and flow rate was maintained at 200 to 300 cells/s. Green fluorescence was detected in FL1 while red fluorescence was detected in FL3. For the SCSA, both FL1 and FL3 photodetectors were used. AO is characterized to depict green fluorescence when it intercalates into native double stranded DNA, and red fluorescence if DNA is single stranded. The amount of red and green fluorescence emitted was measured on a total of 5000 spermatozoa per sample, allowing the calculation of the DNA fragmentation index (\%DFI). DFI is given by the ratio of cells with single stranded DNA (ss DNA) to total cells (ss DNA and ds DNA), reflecting the loss of sperm DNA (Evenson, Larson et al. 2002).

\section{Staining for spermatozoa detection, evaluation of subtle membrane changes/viability and production of reactive oxygen species- multiparametric analysis}

A concentration of $5 \times 10^{6} \mathrm{spz} / \mathrm{mL}$ was obtained by adding $100 \mu \mathrm{L}$ of semen to $900 \mu \mathrm{L}$ of PBS. Multiparametric analysis was performed in a MACSQuant Analyzer (Miltenyi Biotec, Bergisch Gladbach, Germany). Non-sperm events were eliminated after staining of spermatozoa with $0.3 \mu \mathrm{L}$ of a $1.62 \mathrm{mM}$ solution of Hoechst 33342 (Sigma-Aldrich Corporation, St.Louis, MO, USA), which allows the gating of the sperm population (Em $461 \mathrm{~nm})$ after excitation at $350 \mathrm{~nm}$. CellROX Deep Red Reagent (Molecular Probes Europe, Leiden, The Netherlands) allowed measurement of reactive oxygen species (ROS) on the cytoplasm of live and dead cells (identified by Ethidium Homodimer-1). This probe is non-fluorescent in a reduced state, exhibiting, upon oxidation, excitation on $640 \mathrm{~nm}$ and emission on $665 \mathrm{~nm}$ (far-red). Addition of $1 \mu \mathrm{L}$ of a $2.5 \mathrm{mM}$ solution to the sample already marked with Hoechst 33342 , allowed the quantification of ROS on the cells. Samples were incubated for 25 minutes in the dark, at room temperature. The excess of dye was then removed by centrifugation during 5 minutes at $600 \mathrm{x} \mathrm{g}$, followed by the addition of YO-PRO-1 and Ethidium Homodimer -1. Two stock solutions in DMSO were prepared: YO-PRO-1 $(25 \mu \mathrm{M})$ and Ethidium Homodimer-1 $(1.167 \mathrm{mM})$ (Molecular Probes Europe, Leiden, The Netherlands), both excited at $488 \mathrm{~nm}$. To each tube, $1 \mu \mathrm{L}$ of YOPRO-1 and $0.3 \mu \mathrm{L}$ of Ethidium Homodimer-1, and after mixing, all samples were incubated in dark at room temperature for 5 minutes. This staining procedure distinguishes four sperm subpopulations. The first is the subpopulation of unstained live spermatozoa without any membrane alteration. The second subpopulation is correspondent to YO-PRO-1 positive cells, that is, emitting green fluorescence $(509 \mathrm{~nm})$, and bearers of slight alterations on membrane permeability characteristic of early stages of apoptosis. On this stage, cells are not permeable to Ethidium Homodimer-1 dye. This is not the case on the other two subpopulations, the first one composed by spermatozoa stained with both dyes (showing green and red fluorescence), characteristic of early necrosis stages, and the last one, characterized only by red fluorescence, that is Ethidium Homodimer-1 positive $(617 \mathrm{~nm})$, corresponding to late necrotic spermatozoa. Early membrane changes and sperm viability were assessed after gating with Hoechst 33342 which eliminated non-cellular events (debris).

\section{Detection of mitochondrial membrane potential}

The dye 5,5',6,6'-tetrachloro 1,1', 3,3' tetraethylbenzymidazolyl carbocianine iodine (JC-1, Molecular Probes Europe, Leiden, The Netherlands) is a lipophilic cationic compound that has the ability of crossing the plasmalemma and label mitochondria. It has the specificity of marking mitochondria with high membrane potential, by forming multimeric aggregates which emit in the $590 \mathrm{~nm}$ orange wavelength. On the other hand, when in presence of mitochondria with low membrane potential, JC-1 forms monomers emitting green in the 525-530 nm wavelength. Both emissions occur after excitation on $488 \mathrm{~nm}$. After preparation of a $3 \mathrm{mM}$ stock solution of JC-1 in DMSO, $0.5 \mu \mathrm{L}$ of it was added to a sperm suspension of $5 \times 10^{6}$ spermatozoa in one $\mathrm{mL}$ of PBS. Sperm samples were incubated for 40 minutes in the dark, at $37^{\circ} \mathrm{C}$ and measured with Coulter EPICS XL (Coulter Corporation Inc., Miami, FL, USA).

\section{SCSA}


An aliquot of spermatozoa $\left(2 \times 10^{6} \mathrm{spz}\right)$ in $500 \mu \mathrm{L}$ of PBS was frozen at $-20^{\circ} \mathrm{C}$, during a maximum period of one month. After thawing, $1 \mathrm{~mL}$ of TNE $(0,001 \mathrm{M}$ EDTA disodium, $0.001 \mathrm{M}$ Tris $\mathrm{HCl}, 0.15 \mathrm{M} \mathrm{NaCl}, \mathrm{pH} 6.8$ ) was added. After, to $200 \mu \mathrm{L}$ of this solution, $400 \mu \mathrm{L}$ of a Triton solution $(0.15 \mathrm{~N} \mathrm{NaCl}, 0.08 \mathrm{~N} \mathrm{HCl}, 0.1 \%$ Triton $\mathrm{X}-100, \mathrm{pH} 1.4)$ was added and incubation made at room temperature for 30 seconds. At last, $1.2 \mathrm{~mL}$ of an Acridine Orange solution $(0.1 \mathrm{M}$ Citric Acid, $0.2 \mathrm{M} \mathrm{Na}_{2} \mathrm{HPO}_{4}, 1 \mathrm{mM}$ EDTA disodium, $0.15 \mathrm{M} \mathrm{NaCl}, \mathrm{pH}$ 6), incubated for 3 minutes at room temperature in the dark, and measurement performed with the flow cytometer (Ex 502nm Em 525/650nm).

\section{Statistical analysis}

Results were expressed by mean \pm SEM. Data were first examined using the KolmogorovSmirnov test to determine their distribution. As most of the gathered data had a non-Gaussian distribution, a multivariate analysis of variance was performed (ANOVA). When significant differences were found, the non-parametric Mann-Whitney U-test was used. All analyses were performed using SPSS version 17.0 for Windows (SPSS Inc., Chicago, IL, USA). Power analysis was set at 0.8 and significance at $\mathrm{P} \leq 0.05$ and $\mathrm{P} \leq 0.01$.

\section{Results}

\section{Sperm motility and kinematics}

Total motility and progressive motility decreased after the sorting procedure (Figure 1). Nevertheless, both kinematic parameters were similar when comparing the two sorting protocols. Sex-sorting of stallion sperm after a $20 \mathrm{~h}$ storage period at $4^{\circ} \mathrm{C}$ does not decrease total nor progressive motility of spermatozoa. Circular velocity (VCL) decreased after flow sorting on both protocols, from approximately $100 \mu \mathrm{m} / \mathrm{s}$ to $80 \mu \mathrm{m} / \mathrm{s}$ (Figure 2). No significant changes were found on average velocity (VAP) and straight line velocity (VSL). When comparing fresh sorted with refrigerated sorted spermatozoa, the three velocities were similar.

\section{Viability and membrane integrity}

A non-significant decrease on intact spermatozoa was registered after sex-sorting, and a significant increase on the YO-PRO-1 positive subpopulation. As seen on Figure 3, the same increase was present, when comparing fresh sorted (SEX-1) and refrigerated sorted (SEX-2). No significant differences were present between both sorted samples.

\section{Production of reactive oxygen species}

After sorting, there was a noticeable increase on ROS production by live cells (Figure 4). The amount of ROS produced by fresh sorted spermatozoa and refrigerated sorted spermatozoa was approximately the same (46 \pm 10.1 on SEX-1 and $45.9 \pm 9.1$ on SEX-2).

\section{Mitochondrial membrane potential}

An increase on the subpopulation of spermatozoa with low membrane potential (green fluorescence emitted by JC-1) was patent after flow-sorting. No significant changes were present between both sorted samples, as presented on Table 1 .

\section{Sperm Chromatin Structure Assay}

DNA fragmentation index of sorted spermatozoa was significantly higher than DFI of presorted spermatozoa. An increase of approximately $15 \%$ on DFI was present after flow 
cytometric sex-sorting on both fresh (SEX-1) and refrigerated (SEX-2) sorted spermatozoa, as displayed on Figure 5.

\section{Discussion}

We compared two staining protocols for flow-cytometric sex-sorting of stallion spermatozoa. The traditional protocol of sorting fresh semen after 90 minutes of incubation in Hoechst 33342, and sorting of previously stained-cooled semen. Five different parameters were evaluated: motility and velocities, viability and permeability of the membrane, mitochondrial membrane potential, reactive oxygen species and DNA fragmentation index by the SCSA. Interesting results were obtained since no significant differences were found between sorted semen obtained with both protocols suggesting the feasibility of the alternative protocol.

A theory similar to ours was tested by Lindsey et al in stallion spermatozoa. They compared $5^{\circ} \mathrm{C}$ and $15^{\circ} \mathrm{C}$ storage for $18 \mathrm{~h}$, prior to sorting, where apparently higher pregnancy rates were obtained after hysteroscopic insemination of semen stored at $15^{\circ} \mathrm{C}$ (Lindsey, Varner et al. 2005). However, in this study KMT (Kenney Modified Tyrodes) extender was used to store spermatozoa, while in our study a INRA96 modified extender replaced the Kenney extender (Balao da Silva, Macias-Garcia et al. 2012) was used; this extender could provide some extra protective effect over the cold shock as recently demonstrated in our laboratory(Gallardo Bolaños et al, 2014). We decided to use regular refrigeration temperature $\left(4^{\circ} \mathrm{C}\right)$, recognized for the maintenance of motility and fertility after large storage periods (Aurich 2008). This would consist on a new protocol, since on the previous work of pre-sorting refrigerated stallion semen, incubation in Hoechst 33342 was only accomplished after the overnight refrigeration (Lindsey, Varner et al. 2005), while in our work staining took place during refrigeration. The success of this new staining protocol would allow stained semen to be sent on the previous day, sorted and inseminated on the next one.

Significant differences were found between non-sorted and flow sorted stallion sperm on total motility and progressive motility, as seen on Figure 1. However, if we compare semen stained and sexed following the traditional protocol versus semen stained overnight and sexed on the next day, similar motilities were obtained. On the kinematic parameters VCL, VAP and VSL (curvilinear velocity, average path velocity and straightline velocity), slight but non-significant decreases were present post sorting, but the same values when comparing both staining-sorting protocols (Figure 2). The decrease found between velocities of non-sorted and sorted spermatozoa is in agreement with previous published studies (Mari, Rizzato et al. 2010; Balao da Silva, Ortega Ferrusola et al. 2013).

There was an increase in membrane permeability of sex-sorted spermatozoa, as reported in previous studies (Balao da Silva, Ortega Ferrusola et al. 2013). However, the same values of YO-PRO-1 positivity were obtained on both staining-sorting protocols (Figure 3), suggesting that the refrigeration period is not predisposing to increased susceptibility to membrane instability during sorting.

Mitochondrial membrane potential was similar between pre and post-sorting samples, on fresh and on refrigerated sperm, noticeable in Table 1. Nevertheless, a significant increase in the intracellular presence of ROS after the flow sorting procedure was detected (Figure 4). Although mitochondria are one of the factors responsible for ROS generation, this is apparently not dependent from the maintenance of mitochondrial membrane potential (Koppers, De Iuliis et al. 2008; Aitken, Gibb et al. 2012). Previous reports for increased oxidative stress after sorting in other species have been done (Klinc and Rath 2007; Leahy, Celi et al. 2010). The oxidative process could be related to modifications on the plasmalemma and intracellular homeostasis (Oehninger, Blackmore et al. 1995), which is apparently the case on sorted semen (Balao da Silva, Ortega Ferrusola et al. 2013). It is interesting to notice that approximately the same production of ROS was present after both staining protocols (Figure 4), that is, coolingstaining of semen pre-sorting does not cause additional oxidative stress.

In parallel to the increase on ROS, a significant increase on DFI of both stained-sorted samples was also present (Figure 5). This reinforces the reported relation between the oxidative status of spermatozoa and genomic integrity (De Iuliis, Thomson et al. 2009). Low antioxidant capacity 
of seminal plasma may lead to increased DNA damage in equine sperm (Wnuk, Lewinska et al. 2010). As in sorted samples seminal plasma is completely removed, along with its antioxidant capacity (Ball 2008), this would contribute to the destabilization of the plasmalemma and increased oxidative stress. Our findings were not in accordance to what was obtained on boar semen (De Ambrogi, Spinaci et al. 2006), where sorted spermatozoa yielded lower DFI than control. Interspecies variability on the susceptibility to DNA damage of after sex-sorting has been reported in the past, being stallion spermatozoa particularly susceptible (Gibb, Butler et al. 2013). We found that although genomic damage is increased after the sorting procedure, cooled staining-storage of semen prior to sorting does not alter DFI, and therefore this alternative protocol could be considered. The number of individuals used in our study reduced the individual influence on the results. This is important as a significant stallion effect on postsorting DNA integrity has been described by others (Bochenek, Herjan et al. 2005).

Genomic integrity is of extreme importance for embryonic development (Silva and Gadella 2006), especially when more than $30 \%$ of the DNA of spermatozoa is damaged (Agarwal and Said 2003), which occurred after sorting (Figure 5). Although in previous studies we did not find presence of increased apoptotic markers on sorted spermatozoa (Balao da Silva, Ortega Ferrusola et al. 2013), we speculate that it would occur later on timeline, and that preventing the oxidative damage detected at this point would improve the quality of sorted semen.

We conclude that flow sorting after a $20 \mathrm{~h}$ staining-refrigeration period is possible, since all the spermatic parameters were maintained when comparing with the traditional procedure. This new protocol is a valuable option to stallions housed far from the sex-sorting facility. Stained semen shipped on the previous day can be sorted and inseminated closer on the next day closer to ovulation, increasing the possibilities of having a foal from the desired sex. Addition of antioxidants to the extender or 'catch fluid' could be considered, since this would reduce oxidative stress and probably genomic damage induced to spermatozoa.

\section{References}

Agarwal, A. and T. M. Said (2003). "Role of sperm chromatin abnormalities and DNA damage in male infertility." Hum Reprod Update 9(4): 331-345.

Aitken, R. J., Z. Gibb, et al. (2012). "Sperm motility is lost in vitro as a consequence of mitochondrial free radical production and the generation of electrophilic aldehydes but can be significantly rescued by the presence of nucleophilic thiols." Biol Reprod 87(5): 110.

Aurich, C. (2008). "Recent advances in cooled-semen technology." Anim Reprod Sci 107(3-4): 268-275.

Balao da Silva, C., B. Macias-Garcia, et al. (2012). "Effect of Hoechst 33342 on stallion spermatozoa incubated in KMT or Tyrodes modified INRA96." Anim Reprod Sci 131(34): $165-171$.

Balao da Silva, C. M., C. Ortega Ferrusola, et al. (2013). "Sex sorting increases the permeability of the membrane of stallion spermatozoa." Anim Reprod Sci 138(3-4): 241-251.

Balao da Silva, C. M., M. Spinaci, et al. (2013). "Effect of sex sorting on stallion spermatozoa: Heterologous oocyte binding, tyrosine phosphorylation and acrosome reaction assay." Anim Reprod Sci 141(1-2): 68-74.

Ball, B. A. (2008). "Oxidative stress, osmotic stress and apoptosis: impacts on sperm function and preservation in the horse." Anim Reprod Sci 107(3-4): 257-267.

Bochenek, M., T. Herjan, et al. (2005). Sperm chromatin abnormalities after semen sexing procedure - preliminary results. International Equine Gamete Group. Proceedings of the 18th Workshop of the Havemeyer Foundation, Kuhlungsborn, Germany.

Clulow, J. R., H. Buss, et al. (2008). "Field fertility of sex-sorted and non-sorted frozen-thawed stallion spermatozoa." Anim Reprod Sci 108(3-4): 287-297. 
Clulow, J. R., G. Evans, et al. (2010). "Evaluation of the function of fresh and frozen-thawed sex-sorted and non-sorted stallion spermatozoa using a heterologous oocyte binding assay." Reprod Fertil Dev 22(4): 710-717.

De Ambrogi, M., M. Spinaci, et al. (2006). "Viability and DNA fragmentation in differently sorted boar spermatozoa." Theriogenology 66(8): 1994-2000.

De luliis, G. N., L. K. Thomson, et al. (2009). "DNA damage in human spermatozoa is highly correlated with the efficiency of chromatin remodeling and the formation of 8hydroxy-2'-deoxyguanosine, a marker of oxidative stress." Biol Reprod 81(3): 517-524.

Evenson, D. P., K. L. Larson, et al. (2002). "Sperm chromatin structure assay: its clinical use for detecting sperm DNA fragmentation in male infertility and comparisons with other techniques." J Androl 23(1): 25-43.

Gallardo Bolanos, J. M., A. Miro Moran, et al. (2012). "Autophagy and apoptosis have a role in the survival or death of stallion spermatozoa during conservation in refrigeration." PLoS One 7(1): e30688.

Gallardo Bolaños JM, Miró Morán A, Balao da Silva CM, Plaza Dávila M, Martín Muñoz P, Aparicio IM, Tapia JA, Ortega Ferrusola C, Peña FJ 2014 During cooled storage the extender influences processed autophagy marker light chain 3 (LC3B) of stallion spermatozoa Anim Reprod Science 145: 40-46

Garner, D. L. (2001). "Sex-Sorting mammalian sperm: concept to application in animals." J Androl 22(4): 519-526.

Gibb, Z., T. J. Butler, et al. (2013). "Quercetin improves the postthaw characteristics of cryopreserved sex-sorted and nonsorted stallion sperm." Theriogenology 79(6): 10011009.

Klinc, P. and D. Rath (2007). "Reduction of oxidative stress in bovine spermatozoa during flow cytometric sorting." Reprod Domest Anim 42(1): 63-67.

Koppers, A. J., G. N. De luliis, et al. (2008). "Significance of mitochondrial reactive oxygen species in the generation of oxidative stress in spermatozoa." J Clin Endocrinol Metab 93(8): 3199-3207.

Leahy, T., P. Celi, et al. (2010). "Flow-sorted ram spermatozoa are highly susceptible to hydrogen peroxide damage but are protected by seminal plasma and catalase." Reprod Fertil Dev 22(7): 1131-1140.

Lindsey, A. C., D. D. Varner, et al. (2005). "Hysteroscopic or rectally guided, deep-uterine insemination of mares with spermatozoa stored $18 \mathrm{~h}$ at either 5 degrees $\mathrm{C}$ or 15 degrees C prior to flow-cytometric sorting." Anim Reprod Sci 85(1-2): 125-130.

Mari, G., G. Rizzato, et al. (2010). "Quality and fertilizing ability in vivo of sex-sorted stallion spermatozoa." Reprod Domest Anim 45(2): 331-335.

Maxwell, W. M., G. Evans, et al. (2004). "Integration of sperm sexing technology into the ART toolbox." Anim Reprod Sci 82-83: 79-95.

Maxwell, W. M. and L. A. Johnson (1999). "Physiology of spermatozoa at high dilution rates: the influence of seminal plasma." Theriogenology 52(8): 1353-1362.

Oehninger, S., P. Blackmore, et al. (1995). "Effects of hydrogen peroxide on human spermatozoa." J Assist Reprod Genet 12(1): 41-47.

Parrilla, I., J. M. Vazquez, et al. (2005). "Influence of storage time on functional capacity of flow cytometrically sex-sorted boar spermatozoa." Theriogenology 64(1): 86-98.

Silva, P. F. and B. M. Gadella (2006). "Detection of damage in mammalian sperm cells." Theriogenology 65(5): 958-978.

Spinaci, M., C. Vallorani, et al. (2010). "Effect of liquid storage on sorted boar spermatozoa." Theriogenology 74(5): 741-748.

Suh, T. K., J. L. Schenk, et al. (2005). "High pressure flow cytometric sorting damages sperm." Theriogenology 64(5): 1035-1048.

Vidament, M. (2005). "French field results (1985-2005) on factors affecting fertility of frozen stallion semen." Anim Reprod Sci 89(1-4): 115-136. 
Wnuk, M., A. Lewinska, et al. (2010). "Redox status of equine seminal plasma reflects the pattern and magnitude of DNA damage in sperm cells." Theriogenology 74(9): 16771684.

Table 1. Mitochondrial membrane potential of control samples, as well as fresh and refrigerated sorted samples (SEX-1 and SEX-2, respectively), mean \pm SEM. Spermatozoa stained with JC-1, which depicts orange fluoresce when in presence of mitochondria with high membrane potential, and green fluorescence when mitochondria with low membrane potential are present. Both colors are seen when both mitochondria of high and low membrane potential are present. $\mathrm{P}^{\mathrm{a}-\mathrm{b}}<0.05$.

\begin{tabular}{llll}
\hline & High & Intermediate & Low \\
\hline CONTROL-1 & $55,4 \pm 16,7$ & $23 \pm 7,2$ & $21,6 \pm 16,2$ \\
CONTROL-2 & $59,6 \pm 16,7$ & $15,8 \pm 7,2$ & $24,6 \pm 16,2$ \\
SEX-1 & $22,5 \pm 16,7$ & $27,2 \pm 7,2$ & $50,1 \pm 16,2$ \\
SEX-2 & $34,2 \pm 16,7$ & $27,2 \pm 7,2$ & $48,5 \pm 16,2$ \\
\hline
\end{tabular}

\section{Figure Legends}

Fig. 1. Total motility (TM) and progressive motility (PM) of control samples just before entering the flow sorter, fresh sorted stallion spermatozoa (SEX-1) and refrigerated sorted stallion spermatozoa (SEX-2). Evaluation by computer assisted sperm analysis (CASA), mean \pm SEM. Comparisons are made between both sorted samples. $\mathrm{P}^{\mathrm{a}-\mathrm{b}}<0.05$. 
Fig. 2. Spermatic velocities evaluated by computer assisted sperm analysis (CASA) in $\mu \mathrm{m} / \mathrm{s}$ : circular velocity (VCL), average velocity (VAP) and straight line velocity (VSL) of stallion spermatozoa. Measurements made before (CONTROL-1 and CONTROL-2) and after fresh (SEX-1) or refrigerated (SEX-2) semen passage through the flow sorter (mean \pm SEM). Comparisons made between both sorted samples. $\mathrm{P}^{\mathrm{a}-\mathrm{b}}<0.05$.

Fig. 3. Evaluation of viability and membrane permeability of control samples (CONTROL-1 fresh and CONTROL-2 refrigerated) and sorted samples (SEX-1 fresh and SEX-2 refrigerated), stained with YOPRO-1 and Ethidium Homodimer. Intact non-stained cells, negative to both dyes; YoPro-1 spermatozoa have an increase on the membrane permeability; YoPro-1/Eth+ cells are in a late apoptotic status and Eth+ are necrotic spermatozoa. Comparisons made between both sorted samples (mean \pm SEM). $\mathrm{P}^{\mathrm{a}-\mathrm{b}}<0.05$.

Fig. 4. Production of reactive oxygen species (ROS) by spermatozoa after staining with CellROX Deep Red reagent (mean \pm SEM). Ethidium Homodimer allows differentiation between live and dead sperm producing high amounts of ROS, and therefore emitting infrared fluorescence. Control samples of fresh and refrigerated sperm, as well as fresh sorted and fresh refrigerated spermatozoa were analyzed, and comparisons were made between the last two. $\mathrm{P}^{\mathrm{a}-\mathrm{b}}<0.05$

Fig. 5. DNA fragmentation index (DFI) obtained by Sperm Chromatin Structure Assay (SCSA) of control samples of fresh and refrigerated semen (CONTROL-1 and CONTROL-2), and sorted samples of fresh (SEX-1) and refrigerated semen (SEX-2) (mean \pm SEM). Samples were stained by acridine orange, which depicts green fluorescence when intercalated into double stranded DNA and red fluorescence when DNA is single stranded, allowing calculation of DFI. Comparisons made between SEX-1 and SEX-2. $\mathrm{P}^{\mathrm{a}-\mathrm{b}}<0.05$. 